\title{
Spinal Hemorrhage Leading to Cerebral Vasospasm and Infarction
}

\author{
Yuan $\mathrm{Li}^{\mathrm{a}}$, Yevgeniy Isayev ${ }^{\mathrm{b}}$, Yuebing $\mathrm{Li}^{\mathrm{b}, \mathrm{c}}$
}

\begin{abstract}
Only one case of intracranial arterial vasospasm resulting cerebral infarction was previously described in the setting of spinal hemorrhage. The speculation of such symptomatic vasospasm being caused by an intracranial extension of the spinal hemorrhage has been unproven. Here we describe two patients with extensive spinal hemorrhage demonstrated on spinal magnetic resonance imaging (MRI). During their subsequent course, both patients developed cranial signs that included encephalopathy, visual deficit and aphasia. In both cases, small amount of intracranial hemorrhage was detected on head imaging, representing an extension of the spinal hemorrhage. Multiple areas of acute ischemia were seen on brain MRI in both patients. On digital subtraction angiography, diffuse vasospasm of carotid and vertebrobasilar arteries was seen which became normalized prior to discharge. Our report suggests that in treating patients with spinal hemorrhage, physicians need to be aware of possible intracranial hemorrhagic extension and subsequent cerebral vasospasm leading to ischemia. As symptoms of intracranial origin are commonly seen in patients with spinal hemorrhage, the incidence of cerebral vasospasm in this setting could be underestimated.
\end{abstract}

Keywords: Spinal hemorrhage; Intracranial arterial vasospasm; Infarction

Manuscript accepted for publication August 6, 2012

${ }^{a}$ Department of Medicine, Lehigh Valley Health Network, 1250 South Cedar Crest Boulevard, Suite 405, Allentown, PA, United States

${ }^{b}$ Division of Neurology, Department of Medicine, Lehigh Valley Health

Network, 1250 South Cedar Crest Boulevard, Suite 405, Allentown, PA, United States

${ }^{\mathrm{c}}$ Corresponding author: Yuebing Li, Division of Neurology, Department of Medicine, Lehigh Valley Health Network, 1250 South Cedar Crest Boulevard, Suite 405, Allentown, PA, 18103, United States.

Email: Yuebing.Li@lvhn.org

doi: http://dx.doi.org/10.4021/jnr119w

\section{Introduction}

The complication of cerebral vasospasm causing ischemic infarction is commonly observed in the setting of intracranial subarachnoid hemorrhage (SAH). On the contrary, a similar complication secondary to spinal hemorrhage is extremely rare. We performed an exhaustive literature search and found only one case report describing the occurrence of cerebral vasospasm following spinal hemorrhage [1]. Here we report two cases of diffuse cerebral vasospasm resulting in cerebral infarctions where each patient initially developed a spinal hemorrhage with subtle intracranial extension. We discuss the potential pathogenesis and implication of this observation.

\section{Case Report}

\section{Case 1}

A 62 year-old female with a history of cavernous angioma removed from the T8 spinal level two years ago presented with acute pain in the neck, upper back and abdomen. At an outside hospital, magnetic resonance imaging (MRI) of the cervical spine did not detect abnormalities. Upon transferring to our institution on the 11th day of her illness, she was noted to have neck stiffness and bilateral leg weakness. Her level of consciousness was normal, and head CT was negative for acute ischemia or hemorrhage. A lumbar puncture showed bloody cerebrospinal fluid (CSF) with a red blood cell count of $754,000 / \mathrm{mm}^{3}$ and a protein level of $1440 \mathrm{mg} /$ dl. Spinal MRI revealed the presence of extensive subarachnoid hemorrhage in the thoracic and lumbar spinal segments (Fig. 1). A hemorrhagic intradural extramedullary mass was detected at the upper thoracic level which was felt to represent an arteriovenous malformation. On day 14 she exhibited signs of decreased alertness and poor vision. Brain MRI revealed diffuse acute ischemia in the bilateral posterior frontal and parieto-occipital lobes. Increased T2 signals were detected in the cerebral subarachnoid space suggesting the presence of high CSF protein content or subarachnoid hemorrhage. On day 15 , repeat head CT verified the presence 


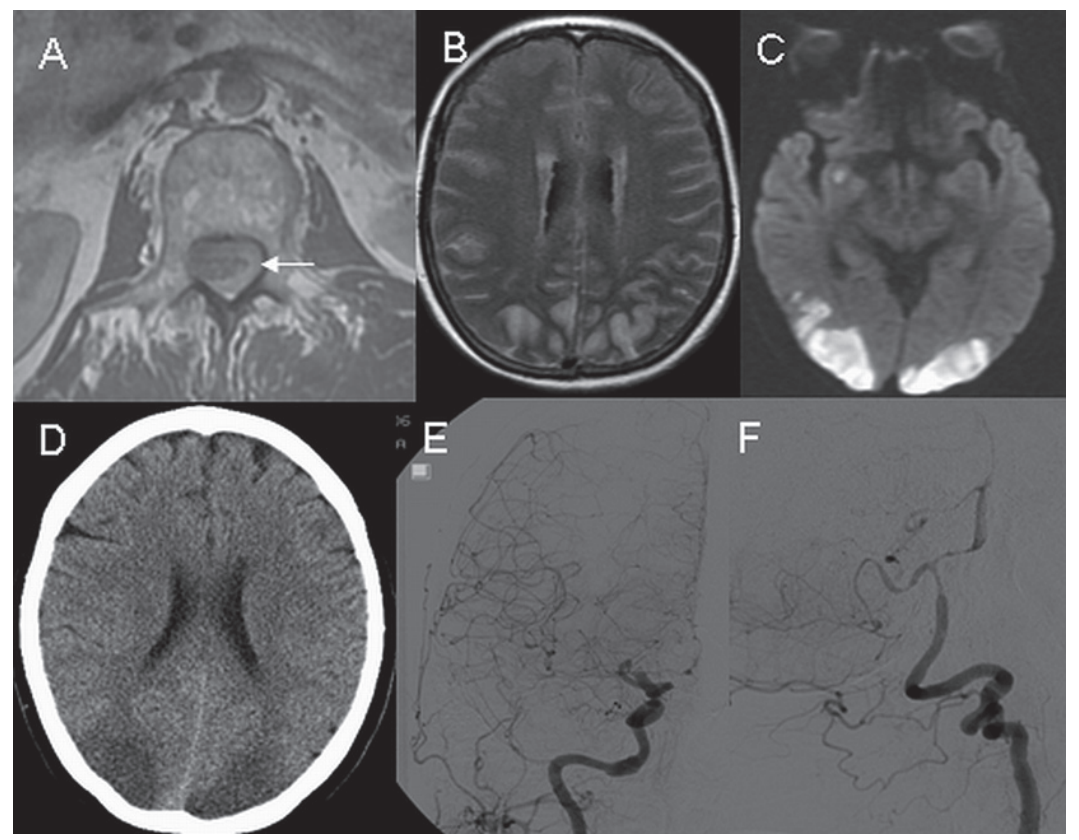

Figure 1. Axial T1- weighted MRI of the upper lumbar spine on day 11 shows a spinal hemorrhage (A, arrow). On day 14 , axial MRI images of the head reveal diffusely increased T2 signals in the subarachnoid space (B) and presence of ischemia on diffusion-weighted imaging (C). On day 15, non-contrast CT scan of head reveals ischemia without hemorrhage (D), and digital subtraction angiography demonstrates diffuse narrowing of the carotid (E) and vertebrobasilar $(F)$ arteries.

of ischemia in the above regions without detectable intracranial hemorrhage. Digital subtraction angiography (DSA) revealed diffuse narrowing of bilateral anterior, middle, posterior cerebral and vertebrobasilar arteries (Fig. 1). On day 19, brain MRI showed a resolution of previously noted abnormal T2 signals in the subarachnoid space but areas of ischemia persisted. On day 31, cerebral CT angiogram revealed a complete resolution of vasospasm (data not shown). At discharge, patient had cortical blindness and bilateral leg weakness, both of which improved minimally over the next four years.

\section{Case 2}

A 73 year-old female was admitted with a two-day history of acute severe upper back pain. On the 5th day of her illness, she developed acute bilateral lower extremity weakness. MRI showed extensive subdural/subarachnoid hemorrhage in the thoracic and lumbar regions (Fig. 2). On day 7 she underwent a thoracic spine exploration, during which extensive subdural and subarachnoid hemorrhage was evacuated. Intra-operatively, a hemorrhagic dural arteriovenous fistula was identified and the feeding artery coagulated. On day 16 she developed symptoms of encephalopathy and generalized weakness, and head CT showed minimal subarachnoid hemorrhage in the parieto-occipital region that was thought to be secondary to the recently occurring spinal hemorrhage and the evacuation procedure (Fig. 2). On day 20 she developed aphasia and mild right arm weakness, and brain MRI revealed acute ischemia in the left insular cortex, left temporal, left parietal and right temporal lobes (Fig. 2). A transesophageal echocardiogram did not reveal an embolic source for ischemia. On day 22, DSA study showed diffuse narrowing of both anterior and posterior cerebral arteries predominately in the distal branches. No cerebral aneurysm or arteriovenous malformation was found (Fig. 2). On day 33, follow up DSA showed a near-complete resolution of vasospasm. The patient was discharged to rehabilitation with minimal right sided weakness. At three-month followup her neurological examination was normal.

\section{Discussion}

Historically recognized as a rare condition, spinal hemorrhage has an increasing incidence owing to advances in spine imaging, usages of antithrombotic therapy and administrations of spinal procedures. Kreppel, et al. conducted a metaanalysis of 613 patients with spinal hemorrhage of epidural, subdural, subarachnoid and intramedullary subtypes, and summarized the following observation: 1) Most spinal hemorrhages are localized at the cervicothoracic or thoracolumbar junctional level; 2) Clinically, spinal hemorrhage often presents with acute or subacute pain at the level of hemorrhage with variable degree of paraparesis and bladder disturbance; 3) Contributing factors for spinal hemorrhage devel- 


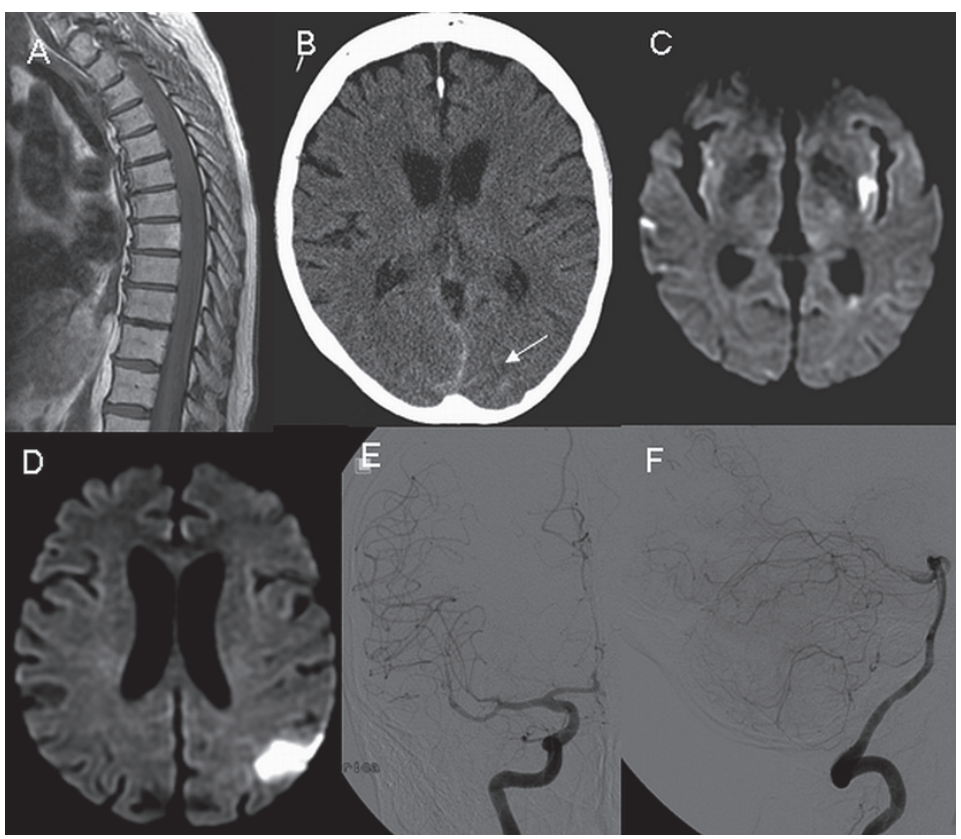

Figure 2. Sagittal T1 weighted MRI of the thoracic spine reveals extensive hemorrhage on day 5 (A). Non-contrast head $C T$ on day 16 shows the presence of minimal amount of subarachnoid hemorrhage (B, arrow). On day 20 , axial diffusion-weighted images of the head demonstrate multiple areas of ischemia ( $C$ and $D)$. Digital subtraction angiography on day 22 demonstrates the presence of diffuse narrowing of the carotid $(E)$ and vertebrobasilar $(F)$ arteries.

opment included trauma, vascular malformation, dissecting aortic aneurysm, coagulopathy, tumor, lumbar puncture, spinal anesthesia and others; 4) Idiopathic spinal hemorrhage without identifiable causes remains as the most common and accounted for approximately $30 \%$ of patients [2]. Traditionally, intracranial and spinal hemorrhages are viewed as separate entities. However, one can lead to the other through the cerebrospinal fluid pathway or other channels.

Cerebral vasospasm secondary to intracranial SAH is well-documented in the literature. Angiographic vasospasm is seen in 30 to $80 \%$ of patients post intracranial aneurysmal SAH, typically observed between 3 and 14 days after the onset. The development of cerebral vasospasm could be related to spasmogenic substances released during the lysis of subarachnoid blood clots that act in close spatial relationship with arterial wall. Proposed mechanisms included vasoconstriction due to accumulation of endothelin, decreased level of nitric oxide or inflammatory responses mediated by oxygen free radicals and cytokines [3]. Early investigations suggested that the amount of hemorrhage in subarachnoid space is a major determinant of cerebral vasospasm. However, further reports indicated that the presence of thick subarachnoid blood is non-essential for the development of cerebral vasospasm. For example, severe vasospasm was observed in patients with minimal hemorrhage in subarachnoid space or in patients with intraventricular hemorrhage while no subarachnoid blood was detected [4]. Vasospasm was also observed following perimesencephalic nonaneurysmal $\mathrm{SAH}$ in which only minimal amount of blood appeared in prepontine and interpeduncular cisterns [5]. In supporting such observations, both our patients developed severe and diffuse vasospasm in the absence of significant intracranial subarachnoid hemorrhage. In both patients, DSA was performed after the diagnosis of cerebral ischemia was already established, therefore DSA was unlikely responsible for the occurrence of underlying vasospasm.

Setzer et al. described a case of cerebral vasospasm resulting in cerebral infarction following spinal hemorrhage and subsequent surgical evacuation in the absence of any detectable intracranial bleeding on imaging. Their speculation was that undetectable hemoglobin of low-concentration entered into the subarachnoid space during hematoma evacuation surgery, traveled to basal cisterns and caused cerebral vasospasm. The relative low concentration of hemorrhage accounted for the negative head imaging [1]. Chaichana and colleagues reported the development of cerebral vasospasm in a case of bacterial meningitis after spinal surgery. In their case, the CSF red blood cell count was elevated at $1800 / \mathrm{mm}^{3}$, suggesting the presence of small amount of subarachnoid hemorrhage that may be responsible for cerebral vasospasm but may not be sufficient for a visualization on head imaging [6]. There have been cases of cerebral vasospasm developing after traumatic lumbar puncture and myelography, possible related to occurrence of undetectable subarachnoid hemorrhage [7]. In our first case, a non-contrast CT of head was negative for intracranial hemorrhage but a subsequent brain MRI showed diffusely increased T2 signals within the intracra- 
nial subarachnoid space suggesting high protein concentration or hemorrhage. We felt that subarachnoid hemorrhage was more likely as these signal changes quickly reversed on subsequent MRI imaging. In our second case, head CT revealed a small amount of intracranial subarachnoid hemorrhage in the posterior regions ten days following a spinal hematoma evacuation. In both cases no other etiologies of intracranial hemorrhage were identified. The source of hemorrhage was most likely secondary to a migration of blood from thoracic spinal level to brain via the subarachnoid space, and our findings verified the previous speculation by Setzer and his colleagues [1].

Symptoms of intracranial origin in patients with spinal hemorrhage have been well documented in the literature. Spinal hemorrhage can be associated with meningismus, disturbances of consciousness, cranial nerve deficits and epileptic seizures. In the meta-analysis by Kreppel et al., 36 of 613 (5.9\%) cases of spinal hemorrhage presented with mainly cerebral signs. In the subgroup of patients with subarachnoid spinal hemorrhage, 34 of 96 (35.4\%) cases showed cerebral symptoms resembling cerebral hemorrhage [3]. Caroscio et al. reviewed another collection of 50 cases of spinal subarachnoid hemorrhage and found that intracranial symptoms or signs were present in $80 \%$ of patients [8]. When present, these symptoms often hide spinal symptoms and lead to misdiagnoses. Since many of these cases did not have vascular studies and some historical cases were described prior to the modern imaging era, intracranial symptoms in a portion of these patients could be secondary to undetected cerebral vasospasm. Therefore, the true incidence of cerebral vasospasm following spinal hemorrhage could be well underestimated.

Our case report has dual implications. First, in treating patients with spinal hemorrhage, physicians need to be aware of the possible vascular complications of intracranial hemorrhagic extension, particularly in those patients with cranial signs. Diffuse vasospasm causing ischemia can occur even if such intracranial hemorrhagic extension is minimally seen on imaging. Secondly, our report also points out a rare cause of cerebral vasospasm from spinal hemorrhage.
In studying patients with cerebral vasospasm without identified etiologies, a careful evaluation and exclusion of spinal hemorrhage is warranted, especially in those patients with spinal signs such as back pain or lower extremity weakness.

\section{References}

1. Setzer M, Weidauer S, Zimmermann M, Berkefeld J, Seifert V, Raabe A. Severe delayed diffuse cerebral vasospasm and cerebral infarctions following spinal subdural hemorrhage. Acta Neurochir (Wien). 2004;146(5):517520 .

2. Kreppel D, Antoniadis G, Seeling W. Spinal hematoma: a literature survey with meta-analysis of 613 patients. Neurosurg Rev. 2003;26(1):1-49.

3. Pradilla G, Chaichana KL, Hoang S, Huang J, Tamargo RJ. Inflammation and cerebral vasospasm after subarachnoid hemorrhage. Neurosurg Clin N Am. 2010;21(2):365-379.

4. Frontera JA, Claassen J, Schmidt JM, Wartenberg KE, Temes R, Connolly ES, Jr., MacDonald RL, et al. Prediction of symptomatic vasospasm after subarachnoid hemorrhage: the modified fisher scale. Neurosurgery. 2006;59(1):21-27; discussion 21-27.

5. Schievink WI, Wijdicks EF, Spetzler RF. Diffuse vasospasm after pretruncal nonaneurysmal subarachnoid hemorrhage. AJNR Am J Neuroradiol. 2000;21(3):521523.

6. Chaichana K, Riley LH, 3rd, Tamargo RJ. Delayed cerebral vasospasm secondary to bacterial meningitis after lumbosacral spinal surgery: case report. Neurosurgery. 2007;60(1):E206-207; discussion E207.

7. Smith RA, Collier HF, Underwood FO. Cerebral vasospasm following myelography. Surg Neurol. 1973;1(2):87-90.

8. Caroscio JT, Brannan T, Budabin M, Huang YP, Yahr MD. Subarachnoid hemorrhage secondary to spinal arteriovenous malformation and aneurysm. Report of a case and review of the literature. Arch Neurol. 1980;37(2):101-103. 